https://helda.helsinki.fi

\title{
The New Wheat Vernalization Response Allele Vrn-D1s is Caused by DNA Transposon Insertion in the First Intron
}

\section{Muterko , Alexandr}

2015-04

Muterko , A , Balashova , I, Cockram , J , Kalendar , R \& Sivolap , Y 2015 , ' The New

Wheat Vernalization Response Allele Vrn-D1s is Caused by DNA Transposon Insertion in the First Intron ' , Plant Molecular Biology Reporter , vol. 33 , no. 2 , pp. 294-303 . https://doi.org/10.1007/s11105-01

http://hdl.handle.net/10138/155664

https://doi.org/10.1007/s11105-014-0750-0

acceptedVersion

Downloaded from Helda, University of Helsinki institutional repository.

This is an electronic reprint of the original article.

This reprint may differ from the original in pagination and typographic detail.

Please cite the original version. 


\title{
The New Wheat Vernalization Response Allele Vrn-D1s is Caused by DNA Transposon Insertion in the First Intron
}

\author{
Alexandr Muterko • Irina Balashova • James Cockram • \\ Ruslan Kalendar • Yuri Sivolap
}

Published online: 18 June 2014

(C) Springer Science+Business Media New York 2014

\begin{abstract}
Vernalization requirement in hexaploid wheat is largely controlled by a series of homoeologous VERNALIZATION (Vrn) genes, Vrn-A1, Vrn-B1, and Vrn$D 1$. The sequence of the promoter area and first intron of Vrn-D1 were analysed in 77 hexaploid accessions, representing five wheat species (Triticum compactum, Triticum sphaerococcum, Triticum spelta, Triticum vavilovii, and Triticum macha) sampled from different ecogeographic areas within 35 countries. Polymorphism was detected in the $V r n-D 1$ promoter, resulting in a new Vrn-D1 haplotype (named here, Hap-8T). Analysis of $V r n-D 1$ intron-1 sequences revealed a novel insertional mutation in a subset of $T$. spelta and T. compactum accessions. This mutant allele was designated $V r n-D 1 s$. Analysis of the $844 \mathrm{bp}$ insertion revealed it to
\end{abstract}

Electronic supplementary material The online version of this article (doi:10.1007/s11105-014-0750-0) contains supplementary material, which is available to authorized users.

\footnotetext{
A. Muterko $(\bowtie) \cdot I$. Balashova $\cdot$ Y. Sivolap

Department of Genomics and Biotechnology, Plant Breeding and Genetics Institute - National Center of Seed and Cultivar Investigation, Ovidiopolskaya Road 3, Odessa 65036, Ukraine e-mail: muterko@gmail.com

I. Balashova

e-mail: ibalash@ukr.net

Y. Sivolap

e-mail: genome2006@mail.ru

J. Cockram

National Institute of Agricultural Botany (NIAB), Huntington Road, Cambridge CB3 0LE, UK

e-mail: james.cockram@niab.com

R. Kalendar

University of Helsinki, Institute of Biotechnology, MTT Plant Genomics Laboratory, Biocentre 3, P.O. Box 65, Viikinkaari 1, Helsinki 00014, Finland

e-mail: ruslan.kalendar@helsinki.fi
}

be a novel transposable DNA element (named DTA_Chimera_KF800714) not previously described in Triticum, belonging to the $h A T$ superfamily. Finally, we describe a PCR-based assay that discriminates the wild-type $v r n$ D1 allele from the $V r n-D 1 s$ allele. Collectively, the work described here highlights the potential of utilizing minor hexaploid wheat species for the identification of novel alleles of agronomic importance.

Keywords Vernalization $\cdot V r n-D 1 \cdot$ Hexaploid wheat Genetic variation $\cdot h A T$ transposon $\cdot$ Flowering time

\section{Introduction}

The adaptability of wheat to a wide range of environmental conditions is largely determined by allelic diversity in genes regulating seasonal growth habit ( $\mathrm{Vrn}$ genes) and photoperiod response (Ppd genes) (Bentley et al. 2013). Allelic variants at the $V r n$ genes, which determine vernalization requirement, are largely responsible for the division of wheat into winter, spring, and facultative types. Previous studies have shown that vernalization response in wheat is controlled by at least four major genetic loci: Vrn1 (a MADS-box transcription factor), $\operatorname{Vrn} 2$ (a zinc-finger CCT domain gene), $\operatorname{Vrn} 3$ (homologous to the Arabidopsis gene FLOWERING LOCUS T), and Vrn4 (uncloned) (Pugsley 1972; Goncharov 2003; Kippes et al. 2014; Yan et al. 2004b, 2006). Under long-day conditions, $\operatorname{Vrn} 2$ and $\operatorname{Vrn} 3$ are expressed (Yan et al. 2004b, 2006). The $V r n$ genes interact epistatically, with $\operatorname{Vrn} 2$ acting as a direct repressor of $\mathrm{Vrn} 3$ which in turn, upregulates $\mathrm{Vrn} 1$ (Distelfeld et al. 2009; Dubcovsky et al. 2008; Trevaskis et al. 2007; Yan et al. 2006). During vernalization, Vrn2 transcription is progressively downregulated (Dubcovsky et al. 2006; Yan et al. 2004b). Once vernalization requirement has been fully met, $\mathrm{Vrn} 2$-mediated repression of $\mathrm{Vrn} 1$ is 
alleviated, with $\operatorname{Vrn} 1$ (or a gene regulated by $\operatorname{Vrn} 1$ ) subsequently maintaining the downregulation of $\mathrm{Vrn} 2$ transcription (Loukoianov et al. 2005). The interactions among Vrn1, Vrn2, and $\operatorname{Vrn} 3$ form a feedback regulatory loop (Distelfeld et al. 2009), in which $\operatorname{Vrn} 3$ is integrator of the vernalization and photoperiod floral pathways (Yan et al. 2006; Trevaskis et al. 2007). Thus, changes in the transcription of any of the Vrn genes in this feedback loop result in the modulation of the expression profiles of the other two genes. It is hypothesized that $V r n-D 4(V r n 4)$ operates upstream (or is a part) of the $V r n 1 /$ $\operatorname{Vrn} 2 / \operatorname{Vrn} 3$ positive feedback loop. However, it is not currently known which of these genes is the potential target of Vrn-D4 (Kippes et al. 2014).

In hexaploid wheat, homoeologous $\mathrm{Vrn} 1$ genes are located on the long arms of the group 5 chromosomes, and named $\mathrm{Vrn}$ $A 1, V r n-B 1$, and Vrn-D1 (Law et al. 1975; Iwaki et al. 2002; Yan et al. 2003). The wheat Vrn1 homoeologues encode MADS-box transcription factors with homology to the Arabidopsis meristem identity genes APETALA1, CAULIFLOWER, and FRUITFULL (Yan et al. 2003; Danyluk et al. 2003; Trevaskis et al. 2003; Kinjo et al. 2012). These genes regulate the transition between vegetative and reproductive phase (Ferrandiz et al. 2000; Shitsukawa et al. 2007). The presence of the dominant $\mathrm{Vrn}$-Al allele results in complete elimination of vernalization requirement (spring type, earliest heading time), whereas the presence of dominant $\mathrm{Vrn}$ $B 1$ or $V r n-D 1$ alleles alone is associated with residual vernalization requirement (facultative type, intermediate, and the later heading time, respectively) (Stelmakh 1993, 1998). These differences in allelic potency have been shown to correlate with their relative expression level (Loukoianov et al. 2005).

Wheat Vrn1 has thought to possess multiple regulatory regions (Distelfeld et al. 2009; Diallo et al. 2012). Dominant spring (vernalization insensitive) $\mathrm{Vrn} 1$ alleles are known to be conferred by mutations in the promoter (Yan et al. 2004a; Golovnina et al. 2010; Pidal et al. 2009; Chu et al. 2011) or intron-1 (Fu et al. 2005; Dubcovsky et al. 2006; Santra et al. 2009; Shcherban et al. 2012). These mutations predominantly represent deletions spanning putative cis-regulatory regions, and often arose by double-stranded DNA break repair via nonhomologous recombination (Cockram et al. 2007b). It is thought that putative cis-regulatory regions in the promoter and intron-1 regulate vernalization response independently (Distelfeld et al. 2009; Zhang et al. 2012).

In previous studies, accessions with spring seasonal growth habit have been identified in wild diploid wheat species such as Triticum boeoticum Boiss, Triticum araraticum Jakubz., and Triticum urartu Thum. (Golovnina et al. 2010). Additionally, there are numerous reports of deletions and insertions within the promoter or intron-1 of $\mathrm{Vrn} 1$ genes in spring cultivars of Triticum aestivum and wild tetraploid wheat (Fu et al. 2005; Dubcovsky et al. 2006; Yan et al. 2004a; Golovnina et al. 2010).
The dominant (vernalization nonresponsive) $V r n-D l a$ allele in T. aestivum has a large deletion within intron-1 and is associated with spring seasonal growth habit (Fu et al. 2005; Yan et al. 2004a). However, no differences have been found in the promoter sequence compared to the recessive $v r n-D 1$ allele. A single nucleotide polymorphism (SNP) has been reported in the predicted CArG-box within the promoter region of $V r n-D 1 b$ alleles found in facultative wheat accessions. However, Vrn-DIb has the same deletion in intron-1 as $V r n-D 1 a$ which decreases vernalization sensitivity (Zhang et al. 2012).

More recently, a large deletion in the first intron of $V r n-D 1$ gene was reported in Aegilops tauschii Coss (the diploid D genome progenitor). This deletion was associated with a lack of vernalization requirement under long-day conditions (photoperiod $>15 \mathrm{~h}$ ), but had no influence on heading date in field conditions (Takumi et al. 2011). However, there are no reports identifying $V r n-D 1$ allelic variants in hexaploid wheat species other than T. aestivum.

In this study, we identify sequence variation at $V r n-D 1$ in five minor hexaploid wheat species (genome AABBDD), such as Triticum compactum, Triticum sphaerococcum, Triticum spelta, Triticum vavilovii, and Triticum macha, define their interspecies distribution and identify new allelic variants. Additionally, a novel Vrn-D1 intron-1 insertion was identified, which represents a member of a new transposon family.

\section{Materials and Methods}

\section{Plant Material}

A total of 77 accessions representing five hexaploid wheat species from 35 countries and different ecogeographic areas were investigated (Supplementary Table 1). These include domesticated hulled wheat (T. spelta L., T. macha Dekap, and $T$. vavilovii Jakubz) and free-threshing wheat (T. compactum Host, T. sphaerococcum Percival). Germplasm was obtained from the National Plant Germplasm System (NPGS, USA) and National Center of Plant Genetic Resources (Ukraine).

\section{DNA Extraction and PCR Amplification}

Total DNA from 4-day-old wheat seedlings was extracted following a modified CTAB-method (Doyle et al. 1987). PCR reactions consisted of DNA ( $\sim 0 \mathrm{ng}$ ), $20 \mathrm{mM}$ Tris- $\mathrm{HCl}$ (pH 8.8), $10 \mathrm{mM}\left(\mathrm{NH}_{2}\right)_{2} \mathrm{SO}_{4}, 2.4 \mathrm{mM} \mathrm{MgCl}, 1 \mathrm{mM} \mathrm{KCl}$, $0.1 \%$ Triton X-100, $250 \mu \mathrm{M}$ dNTPs, $1.5 \%$ DMSO, $0.5 \mu \mathrm{M}$ each primer, and 1.2 U Taq polymerase. A region of $\mathrm{Vrn}-\mathrm{D} 1$ intron-1 was amplified using a primer multiplex consisting of primers Intr1/D/F, Intr1/D/R3, and Intr1/D/R4 (Fu et al. 2005). Additionally, primer pair VRN1INT1_F (Dubcovsky et al. 
2006), Vrn-A1-intr_R1 (this study) were used to further study specific regions of intron-1. A region of the promoter was amplified using previously published primers: VRN1DF and VRN1-INT1R (Yan et al. 2004a). PCR was performed using the following program: denaturation at $94^{\circ} \mathrm{C}(2 \mathrm{~min}) ; 30$ cycles of amplification at $94{ }^{\circ} \mathrm{C}(10 \mathrm{~s})$, annealing $(15 \mathrm{~s}), 74^{\circ} \mathrm{C}(50 \mathrm{~s})$ per cycle; three cycles at annealing $(15 \mathrm{~s}), 74{ }^{\circ} \mathrm{C}(50 \mathrm{~s})$, and a final elongation step of $72{ }^{\circ} \mathrm{C}$ for $3 \mathrm{~min}$. Further details of all primers, including annealing temperatures, are listed in Table 1. Amplification products were separated on $7 \%$ nondenaturing polyacrylamide gels under $13 \mathrm{~V} / \mathrm{cm}$ for $3 \mathrm{~h}$. Visualization of amplification products was conducted using a modified silver staining protocol (Budowle et al. 1991).

Cloning and Sequencing of PCR Fragments

PCR amplicons were separated on $1.5 \%$ agarose gels, gel extracted using a GeneJET Gel Extraction Kit (Thermo Scientific), and DNA fragments ligated into pGEM-T (Promega), following the manufacturer's instructions. Plasmid DNA was transformed into JM109 Escherichia coli competent cells (L2001, Promega). Transformed cells containing the plasmid carrying an insertion of foreign DNA fragment were detected using white-blue selection on growth medium containing ampicillin, X-Gal, and IPTG. Positive colonies were tested for the presence of cloned PCR products by PCR with universal pUC primers (forward and reverse M13 primers), followed by separation and visualization of PCR products on agarose gels. Plasmid DNA was extracted using GeneJET Plasmid Miniprep Kit (Thermo Scientific) and sequencing PCRs performed using an ABI3700 Bioanalyser (Applied Biosystems), following the manufacturer's instructions. Fluorescently labeled extension products were precipitated and resuspended in HiDi (Applied Biosystems).The five partial $V r n-D 1$ sequences reported in this paper were deposited in GenBank under accessions: KF939526-KF939529 (promoter area), KF800714 (intron-1).

Data Analyses

DNA sequence searches of the T. aestivum genome were performed using BLASTn (Zhang et al. 2000) searches of NCBI databases (http://www.ncbi.nlm.nih.gov/), project: "454 pyrosequencing of the Triticum aestivum (bread wheat) genome to 5X coverage" (Brenchley et al. 2012) (without runs accessions ERR: 16487-16503, 16576, and 16577). Transposable elements were identified by BLASTn analysis of the online databases, TREP (Anderson et al. 2005, http://wheat. pw.usda.gov/ITMI/Repeats/) and RepBase (Kohany et al. 2006). For in silico PCR, SNP analysis, DNA repeats searches, and analysis of TIRs-TSDs (terminal inverted repeats-target site duplications) associations, computer programs, and scripts were developed. $\mathrm{CpG}$ islands were identified using Geneious 4.8.4 (http://www.geneious.com). Multiple sequence alignments were generated using Clustal W (Thompson et al. 1994).

\section{Results}

Polymorphism Within the Vrn-D1 Promoter

Primer pair VRN1DF/VRN1-INT1R flanks of the promoter area (from -772 to $0 \mathrm{bp}$ ), exon-1 and part of the first intron $(+185$ to $+248 \mathrm{bp})$. The forward primer was designed to be specific to the D genome, while the reverse primer was

Table 1 Vrnl PCR primer details

\begin{tabular}{|c|c|c|c|c|c|}
\hline Primers & Primer sequence $\left(5^{\prime}-3^{\prime}\right)$ & $\begin{array}{l}\text { Annealing temp. } \\
\left({ }^{\circ} \mathrm{C}\right)\end{array}$ & $\begin{array}{l}\text { Expected PCR } \\
\text { product size (bp) }\end{array}$ & Allele & Reference \\
\hline $\operatorname{Intr} 1 / \mathrm{D} / \mathrm{F}$ & gttgtctgectcatcaaatcc & 64 & 997 & $v r n-D 1$ & Fu et al. (2005) \\
\hline Intr1/D/R4 & aaatgaaaaggaacgagagcg & & 1,841 & $V r n-D 1 s$ & \\
\hline Intr1/D/F & gttgtctgcctcatcaaatcc & 64 & 1,671 & Vrn-Dla & \\
\hline Intr1/D/R3 & ggtcactggtggtctgtgc & & & & \\
\hline VRN1INT1_F & atgaaactccggcatgaaga & 61 & 837 & $v r n-D 1$ & Dubcovsky et al. (2006) \\
\hline \multirow[t]{4}{*}{ Vrn-A1-intr_R1 } & cttgtccecgtgagctacttac & & 841 & $v r n-A I^{\mathrm{a}}$ & This study \\
\hline & & & 1,336 & $V r n-A l f$ & \\
\hline & & & 1,345 & $V r n-A 1 h$ & \\
\hline & & & 1,681 & $V r n-D 1 s$ & \\
\hline VRN1DF & cgacccgggcggcacgagtg & 61 & 1,012 & Promoter area of $v r n-D 1$ gene & Yan et al. (2004a) \\
\hline \multirow[t]{3}{*}{ VRN1-INT1R } & gcaggaaatcgaaatcgaag & & 1,018 & & \\
\hline & & & 1,019 & & \\
\hline & & & 1,020 & & \\
\hline
\end{tabular}

${ }^{\mathrm{a}}$ Intact intron-1 
previously designed to amplify $V r n-A 1$ (Yan et al. 2004a). Sequence alignment indicated that the reverse primer anneals to $V r n 1$ genes from all three genomes (A, B, and D). Accordingly, this primer combination was optimized for investigation of $V r n-D 1$ (Table 1). PAA electrophoresis of the resulting PCR amplicons from the 77 hexaploid wheat accessions studied here (Supplementary Table 1) identified two amplicon sizes-a 1,020-bp fragment (as predicted from the reference $V r n-D 1$ genomic sequence) and a 1,019-bp fragment (Fig. 1a). The smaller PCR product was amplified from four T. spelta accessions (UA0300304, PI 272573, PI 306550 , and PI 323438), as well as from one T. macha accession (PI 428178). PCR amplicons from four accessions were cloned and fully sequenced (GenBank: KF939526KF939529).

Multiple alignments of these sequences with $V r n-D 1$ reference sequences (GenBank: AB630962, AB630964, and AY616454) showed several polymorphic features. To further investigate this region of $V r n-D 1$, in silico PCR using GenBank, EMBL, DDBJ, and RefsSeq databases was conducted. In silico amplicons of length 1,012, 1,018, and $1,020 \mathrm{bp}$ for A. tauschii and 1,020 bp for T. aestivum were identified (Supplementary Fig. S1). Among the 1,020-bp amplicons, eight SNPs were detected (G66C, T281C, A394G, C633T, G735A, A747G, G780A, and G868A). However, these polymorphisms were not present in the 1,019-bp amplicons identified here. Additionally, our sequencing did not detect the $8 \mathrm{bp}$ deletion found in the $1,012 \mathrm{bp}$ in silico A. tauschii amplicons. All of the $1,019 \mathrm{bp}$ amplicons identified in this study were characterized by deletion of "C" nucleotides at positions 50 and $52 \mathrm{bp}$, a "T" insertion at 346-site (poly-T region-T-tract) and a "T- > C" mutation at $30 \mathrm{bp}$. It is assumed that these polymorphic features caused the observed differences in amplicon mobility in PAA gels. These mutations were not present in sequences previously found in DDBJ/EMBL/GenBank databases and therefore represent a new $V r n-D 1$ haplotype (designated Hap-8T, T-tract $=(\mathrm{T})_{8}$ whereas an intact sequence $(\mathrm{PCR}$ fragment 1,020 bp) designated Hap-7T, T-tract $\left.=(\mathrm{T})_{7}\right)$.

\section{Discovery of a New Vrn-D1 Allele}

A multiplex PCR with primers Intr1/D/F, Intr1/D/R3, and Intr1/D/R4 was used to investigate a region of intron-1 thought to span a cis-element implicated in the control of vernalization response. Primer pair Intr1/D/F-Intr1/D/R4 spans a region of intron-1 predicted to amplify products of $997 \mathrm{bp}$ in the wildtype (vernalization responsive) allele. However, if the 4,235 bp intron-1 deletion that confers the spring Vrn-Dla allele is present, primer pair Intr1/D/F-Intr1/D/R3 results in amplification of 1,671 bp fragments (Fu et al. 2005).

To examine distribution of $V r n-D 1$ intron-1 allelic variation, multiplex PCR was performed using the 77 accessions that encompass five hexaploid wheat species. Electrophoretic separation of PCR products identified intron-1 length polymorphisms, resolving fragments of 997 bp (identified in 66 accessions, vrn-D1), 1,671 bp (7 accessions, Vrn-Dla), and $1,841 \mathrm{bp}$ (4 accessions, not previously characterized) (Fig. 1b; Supplementary Table 1). This indicates that while carriers of the recessive wild-type winter allele (vrn-D1, $997 \mathrm{bp})$ and dominant spring allele (Vrn-Dla, 1,671 bp) are present in the accessions investigated, a new allelic variant of the $\mathrm{Vrn}$ D1 gene involving a size polymorphism within intron-1 has also been identified $(1,841 \mathrm{bp})$. We subsequently
Fig. 1 PCR amplification of $V r n-1$ genes. $M$ GeneRuler DNA ladder (100-10,000 bp). a Amplification of the promotor region using primers VRN1DF/ VRN1-INT1R-4, 7, and 8 identify the novel haplotype Hap-8T (1,019 bp); Hap-7T (1,020 bp)1-3, 5, 6, and 9-16. b MultiplexPCR targeting

$V r n-D 1$ intron-1-wild type 1-3, 5, 9-11 (no deletion, amplicon= 997 bp); spring Vrn-D1a alleles 6-8 (1,671 bp); novel Vrn-D1s allele (1,841 bp) 4. c Multiplex PCR of the Vrn-D1s and Vrn-Dla accessions targeting $\mathrm{Vrn}-\mathrm{Al}$ and Vrn-D1-vrn-A1/Vrn-D1s 1, 2, and 4 (841/1,681 bp); vrn-A1/VrnD1a-3 (vrn-D1 null allele)

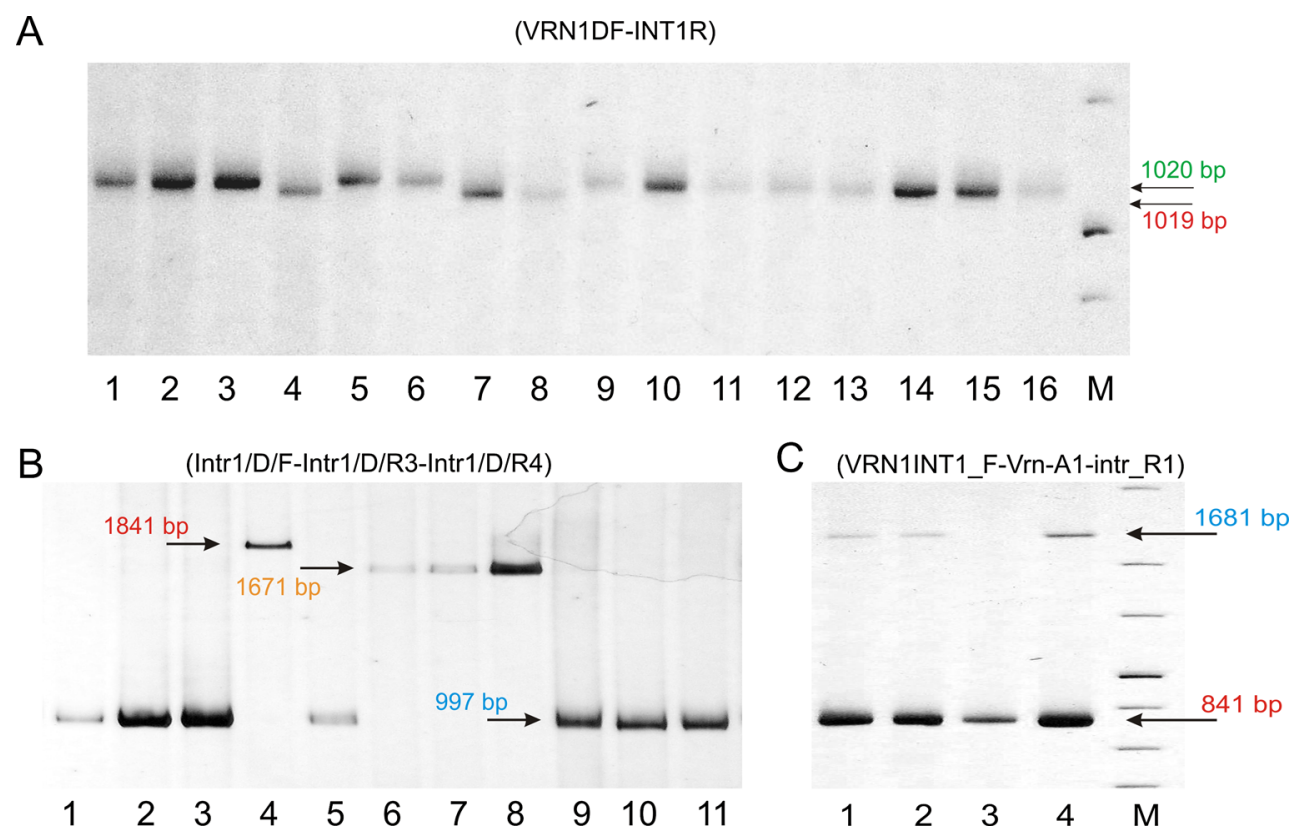


deconvoluted the multiplex design, and performed PCR analysis using two primer pair combinations (Intr1/D/F, Intr1/D/ R3 and Intr1/D/F, Intr1/D/R4). This found the novel 1,841 bp fragment to be amplified by primer pair Intr1/D/F, Intr1/D/R4, indicating that an insertion occurred within intron-1 of the wild-type recessive $v r n-D 1$ allele. Amplicons with length of $1,841 \mathrm{bp}$ from T. spelta (PI 348700) were cloned and sequenced (GenBank accession KF800714). Sequence alignment showed this novel variant includes an 844 bp insertion in $V r n-D 1$ intron-1 (position +1,044; from 859 to $1,702 \mathrm{bp}$, counting from the start of intron-1 in Triple Dirk C (GenBank accession AY747606)). As the novel 1,841 bp amplicon was identified predominantly in $T$. spelta, this new putative $\mathrm{Vrn}$ D1 spring allele was designated $V r n-D 1 s$.

Finally, primer pair VRN1INT1_F (Dubcovsky et al. 2006), Vrn-A1-intr_R1 (this study), for which sequence alignments show anneal to $V r n-A 1$ and $V r n-D 1$, were optimised for use here. This primer combination was found to simultaneously amplify intron-1 regions from both Vrn-A1 and Vrn-D1 homologues (Fig. 1c). PCR amplicons with length of $837 \mathrm{bp}$ identify the wild-type winter $v r n-D 1$ allele (no intron-1 deletion), while the 1,681-bp product identifies the novel $V r n-D 1 s$ allele identified in this study. A product of $841 \mathrm{bp}$ is diagnostic for the absence large $V r n-A 1$ intron-1 deletion present in the $V r n-A 1 c$ allele, while products of size 1,336 or $1,345 \mathrm{bp}$ correspond to spring $V r n-A^{m} 1 f$ and $V r n-A^{m} 1 h$ alleles, respectively. Null alleles (absence of PCR fragments) for one or both of the loci indicate the presence of spring $\mathrm{Vrn}-\mathrm{Alc}$ and/or $\mathrm{Vrn}$ D1a alleles.

Discovery and Classification of a Novel Transposon Family

Sequence analysis of the novel 844 bp $V r n-D 1$ intron-1 insertion identified three regions with high GC content $(>60 \%)$, localized to regions 20-191 bp (high GC region 1 (HGC-1)), 304-510 bp (HGC-2) and 759-844 bp (HGC-3). CpG islands were identified in HGC-1 and HGC-3, but not HGC-2 (Fig. 2). HGC-1 and HGC-3 were also found to contain many repeated sequences (direct, tandem, reverse, and inverted) of various lengths (up to $17 \mathrm{bp}$ ).

The 844-bp insertion was used as a query for BLASTn searches of DDBJ/EMBL/GenBank databases. Regions 1167 and 681-836 bp (which corresponds to HGC-1 and HGC-3, respectively) were found to be present as multiple copies in numerous cereal genomes (Triticum, Aegilips, and Hordeum) with high degree of sequence similarity ( $>75 \%)$. Furthermore, a highly similar sequence $(99 \%$ ) was identified, localized on short arm of T. aestivum chromosome 4D (IWGSC_CSS_4DS_scaff_2292513:596-1432). However, these regions have not been annotated. It has been suggested that HGC-1 and HGC-3 appear to represent parts of transposable DNA element (TE), not previously described in the Triticum genome.
Further sequence analysis of the Vrn-D1 insertion identified a specific motif of $11 \mathrm{bp}$, located at positions $1 \mathrm{bp}$ (in a forward orientation) "TAGGACTGGAC" and $826 \mathrm{bp}$ (inverted orientation) "GTCCAGCCCTA". This short terminal inverted repeat (TIR) is flanked by an 8-bp target site duplication (TSD): "GTGCTGGC" (Figs. 2 and 3). Upstream of the 3'-TIR, sequence analysis identifies a conserved pentameric subterminal repeat (STR): $(\mathrm{GCTCG})_{4}$. However, sequence alignment has shown that 5 -TIR carries an "A" nucleotide instead of " $G$ " at position 5 (5'-TIR-5A and 5'-TIR-5G, respectively) and lacks an adjacent STR (Figs. 2 and 3). Considering the evidence listed above, the Vrn-D1 insertion identified in this study appears to be a compound, hybrid, or transposon. Following standard TE classification criteria (Wicker et al. 2007), this mobile element was annotated as non-autonomous DNA transposon, belonging to the class II, subclass 1, order TIR, and superfamily $h A T$ (designated as DTA_Chimera_KF800714).

Multiple alignment was performed for $>500 T$. aestivum DNA sequences which showed high similarity $(>80 \%)$ and which covered $>50 \%$ of the insertion sequence. Two conserved regions were identified (positions 1-168 and 681836), within which regions of increased sequence similarity were identified: positions $1-11 \mathrm{bp}$ (spanning the $5^{\prime}$-TIR) and $806-836$ bp ( $3^{\prime}$-TIR and STR). The reduced sequence conservation within the intervening region (168-681 bp) indicates the existence of multiple transposon types within the family. Sequence alignment showed the TIRs to possess SNPs (5'TIR-5(A/G) and 3'-TIR-3(C/T)), which appear not to affect the transposition process, as each TIR mutant corresponds to several different TSDs (Fig. 3). Since 5'-TIR and 3'-TIR are mismatched, it is likely that (1) different transposases specific for each TIR are involved or (2) these SNPs are synonymous.

TIRs and TSDs are used as features for the classification of TE families (Wicker et al. 2007). To investigate the TIRs and TSDs within the TE identified here, 80 bp regions at the $5^{\prime}$ and $3^{\prime}$ ends of the element were used for BLASTn searches of the T. aestivum genome. Using a similarity cutoff $>80 \%$ identity, 742 and 745 hits to the $5^{\prime}$ - and $3^{\prime}$-segment were identified, respectively.

In total, 72, 118, 58, 10, 10, and 1 distinct TSDs identified, associated with $5^{\prime}$-TIR-5A, $3^{\prime}$-TIR-3C (the TIRs found in the $V r n-D 1 s$ TE identified here), 5'-TIR-5G, 3'-TIR-3T, 3'-TIR5T, and 5'-TIR-3A, respectively (Supplementary Tables 2 and 3). Thirty-seven TSDs were common for the TIRs identified in the Vrn-D1s TE, and only 28 and 4 for TIR haplotype combinations $5^{\prime}$-TIR-3G,5G/3'-TIR-3C,5C and 5'-TIR$3 \mathrm{G}, 5 \mathrm{~A} / 3^{\prime}$-TIR-3C,5T, respectively, although these pairs are identical TIRs (Table 2). Matching TSDs were not identified for $5^{\prime}$-TIR-5A/5'-TIR-5G, 3'-TIR-3C/3'-TIR-3T (Table 2; Supplementary Table 4). These data indicate that $5^{\prime}$-TIR-5A and 5'-TIR-5G are commonly found in combination with 3'TIR-3C, but that they are probably nonsynonymous for 


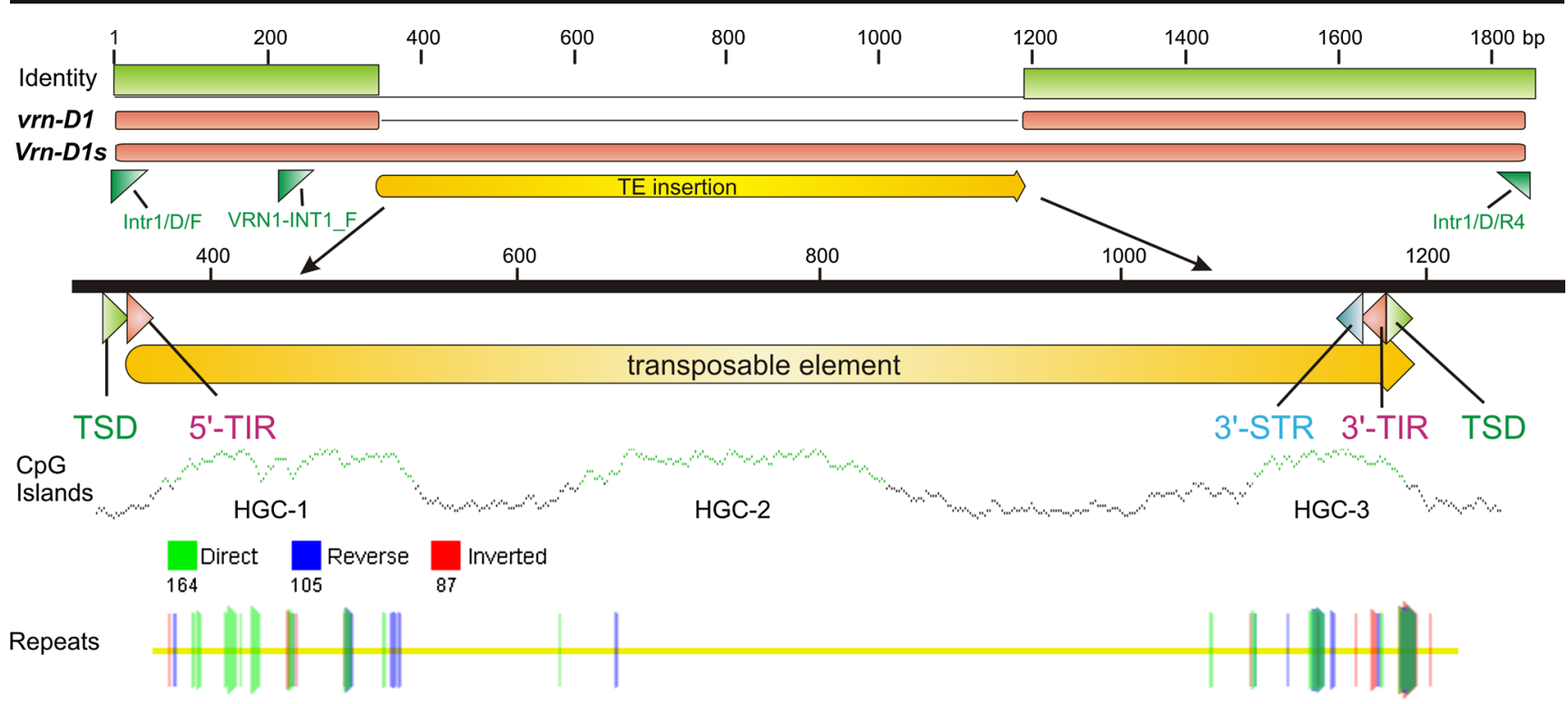

Fig. $2 V r n-D 1$ intron 1, indicating the TE insertion. The wild-type winter allele $(v r n-D I)$ and the novel $V r n-D 1 s$ allele are illustrated, overlaid with TE structure including positions of terminal inverted repeats (TIR), target

transposons of a same family since they do not contain common TSDs, but belong to closely related families. 3'TIR-3C and $3^{\prime}$-TIR-3T in combination with $5^{\prime}$-TIR-5A are nonsynonymous and likely belong to closely related families.

Sequence searches of the TE database RepBase (Kohany et al. 2006) found regions 12-167 bp (encompassing HGC-1) and $684-836$ bp (HGC-3) to be highly homologous ( $>73 \%$ sequence identity) to transposable element belonging to the hAT (hobo/Ac/Tam) superfamily. Regions $12-167$ bp showed $73 \%$ similarity to the maize DNA transposon hAT-N16_ZM, although it lacked regions homologous to the $5^{\prime}$-TIR identified in the Vrn-D1s TE. Regions 681-836 bp showed an overall sequence similarity of $75 \%$ to the Oryza sativa Ac-like nonautonomous DNA transposon, DS-RICE3N. Within this region, the 3'-STR and 3'-TIR showed $85 \%$ similarity to the rice TE, with the wheat $3^{\prime}$-TIR sequence aligning to the first nucleotide position in DS-RICE3N. However, the DS- site duplications (TSD), and subterminal repeats (STR). Distribution of $\mathrm{CpG}$ islands and nucleotide repeats within the TE are also indicated

RICE3N TIR has a two-nucleotide mismatch relative to the wheat $3^{\prime}$-TIR investigated here. Additionally, no common TSDs were identified for these TIRs. Accordingly, we conclude that these transposons belong to different, but closely related families. BLASTn searches of the TREP TE database did not identify any homologous transposons. According to established nomenclature (Anderson et al. 2005), this new family of non-autonomous short transposons with different 5'- and 3'-TIRs was designated, as "Chimera".

\section{Discussion}

Defining allelic variation in hexaploid wheat species related to T. aestivum is of importance as they are used by plant breeders as a source of agronomically valuable traits. For example,

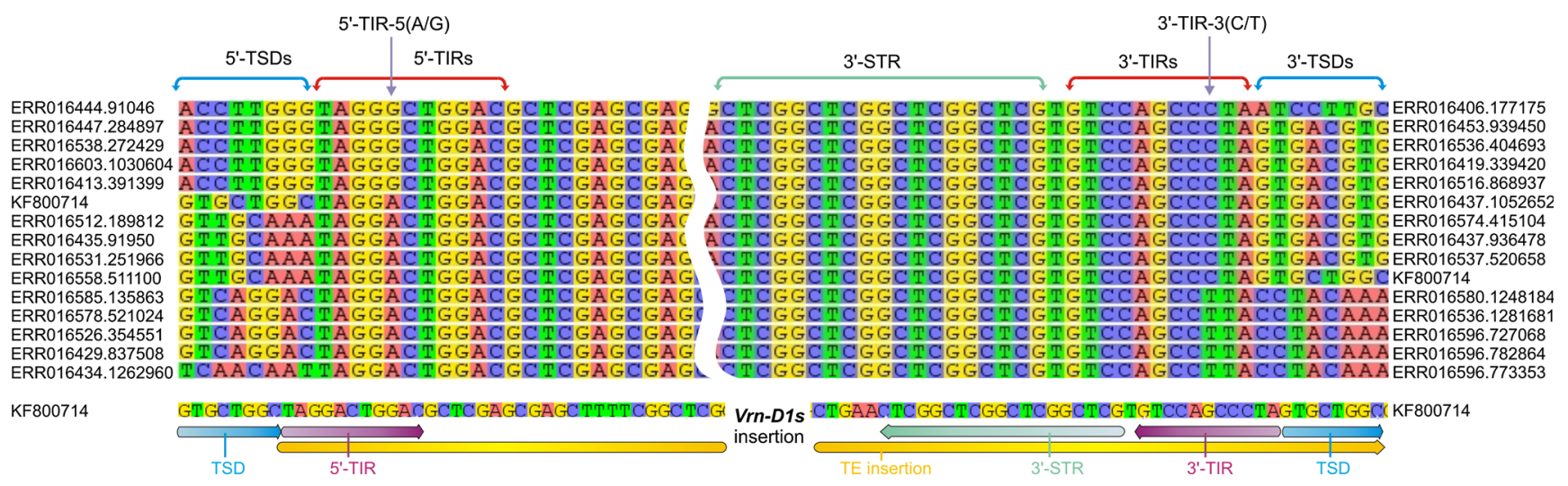

Fig. 3 Multiple alignment of transposons with high sequence ( $80 \mathrm{bp}$ segments) similarity ( $>80 \%)$. The positions of TIRs, TSDs, and SNPs in $5^{\prime}$ - and $3^{\prime}-$ TIRs are indicated 
Table 2 Number of common TSDs for different 5'-TIR/3'-TIR combinations

${ }^{\text {a }}$ Original TIRs (found in the Vrn-D1s TE)

\begin{tabular}{lllllll}
\hline TIRs & $5^{\prime}-3 \mathrm{G}, 5 \mathrm{~A}^{\mathrm{a}}$ & $5^{\prime}-3 \mathrm{G}, 5 \mathrm{G}$ & $5^{\prime}-3 \mathrm{~A}, 5 \mathrm{~A}$ & $3^{\prime}-3{\mathrm{C}, 5 \mathrm{C}^{\mathrm{a}}}^{\prime}$ & $3^{\prime}-3 \mathrm{C}, 5 \mathrm{~T}$ & $3^{\prime}-3 \mathrm{~T}, 5 \mathrm{C}$ \\
\hline $5^{\prime}-3 \mathrm{G}, 5 \mathrm{~A}^{\mathrm{a}}$ & - & - & - & 37 & 4 & - \\
$5^{\prime}-3 \mathrm{G}, 5 \mathrm{G}$ & & - & - & 28 & 2 & 4 \\
$5^{\prime}-3 \mathrm{~A}, 5 \mathrm{~A}$ & & & - & 1 & - & - \\
$3^{\prime}-3 \mathrm{C}, 5 \mathrm{C}^{\mathrm{a}}$ & & & - & - & - \\
3'-3C,5T & & & & & - \\
3'-3 T,5C & & & & & - \\
\hline
\end{tabular}

hexaploid wheat species as T. spelta, T. vavilovii, and T. macha have been identified as potential donors of resistance genes to stripe and leaf rust (Bariana et al. 2002; Gultyaeva et al. 2002), Septoria tritici (Simon et al. 2001), Russian wheat aphid (Nkongolo et al. 1989), Fusarium head blight (Steed et al. 2005; Buerstmayr et al. 2011), etc. Furthermore, systematic investigations may find novel allelic variants at known genes of agricultural importance. In the case of the cereal $\mathrm{Vrn}$ 1 genes, previous surveys of natural variation have identified novel spring and winter alleles, all of which are due to allelic variation within the promotor or intron-1 (Cockram et al. 2007a; Dubcovsky et al. 2006; Fu et al. 2005; Golovnina et al. 2010; Shcherban et al. 2012; Yan et al. 2004a).

For the major vernalization response locus $\operatorname{Vrn}-D 1$, no promoter sequence differences have been reported between dominant $V r n-D 1$ alleles versus recessive $v r n-D 1$ alleles $(\mathrm{Fu}$ et al. 2005; Yan et al. 2004a). However, several mutations (including SNPs and an 8-bp deletion) have previously been identified within winter and spring allelic classes in T. aestivum (Golovnina et al. 2010; Takumi et al. 2011; Zhang et al. 2012) and A. tauschii (Takumi et al. 2011). Just one SNP has been identified associated with later flowering of spring accessions possessing a deletion in intron-1 ( Vrn-D1b allele) (Zhang et al. 2012). In this study of 77 accessions of various hexaploid wheat species, several novel $\operatorname{Vrn}-\mathrm{D} 1$ promoter mutations were identified. However, as none localize to the predicted cis-regulatory regions within the promoter (CArG, VRN, or G boxes), we assume that they do not influence vernalization requirement. PCR fragments of the new Hap-8T haplotype are characterized by increased migration in PAA gels. The observed difference in the amplicon mobility between the Hap-7T and Hap-8T haplotypes cannot be explained by only 1 bp deletion. We assume that the "T" insertion at position $346 \mathrm{bp}$ (thymine-tract region) contributes more to the differences in the electrophoretic mobility between amplicons than other mutations within the Hap-8T haplotype (Supplementary material "T-tract analysis").

Our analysis of $V r n-D 1$ intron-1 revealed a new spring allele $(V r n-D 1 s)$ due to TE insertion, present in three T. spelta accessions (from Spain and England) and one T. compactum accession (Turkey). This indicates that the $V r n-D 1 s$ allele is not specific to a single hexaploid wheat species (T. spelta).
The first intron contains a region required to maintain repression of Vrn1 (von Zitzewitz et al. 2005; Fu et al. 2005; Cockram et al. 2007a; Hemming et al. 2009). Fu at al. (2005) defined a 4.2-kb region of $V r n 1$ intron-1 as the vernalization "critical region". This region (Fig. 4) is defined by the deletions that have a most distal start point (Vrn-Alc of IL369) and most proximal end (the $3^{\prime}$ end of the $V r n-D l a$ deletion) (Fu et al. 2005), within which deletions result in decreased vernalization sensitivity (Fu et al. 2005; Shcherban et al. 2012). It has been hypothesized that the "critical region" contains a binding site for a putative repressor that is downregulated by vernalization (Fu et al. 2005; von Zitzewitz et al. 2005) or encompass a region implicated in maintaining chromatin in a repressive state (Diallo et al. 2012; Oliver et al. 2009). In contrast, the presence of deletions or TE insertion downstream of the "critical region" do not affect vernalization requirement (Fu et al. 2005).

Insertions of TEs in Vrn-Al intron-1 (the dominant Vrn$A^{m} 1 f$ and $V r n-A^{m} 1 h$ alleles) are associated with active expression of $\mathrm{Vrnl}$ in the absence of vernalization, despite being located 200 bp upstream from the proposed "critical region" (Fig. 4) (Dubcovsky et al. 2006). Similarly, TE insertion within this region of barley $\mathrm{Vrn}-\mathrm{H} 1$ intron-1 is also associated with vernalization insensitivity (Cockram et al. 2007a, b). Furthermore, it has been shown in Arabidopsis that intron-1 TE insertion within MADS-box genes can affect regulatory regions adjacent to the insertion point (Liu et al. 2004). Collectively, these results suggest that the "critical region" should be extended further in the $5^{\prime}$ direction.

Mutations within intron-1 of the homoeologous Vrn1 genes are reported to be more effective in suppressing the effect of Vrn2 on flowering time than the allelic variation in the promoter region (Dubcovsky et al. 2008), resulting in the induction of $V r n 1$ independently of $V r n 2$ expression (Dubcovsky et al. 2005; Hemming et al. 2008; Pidal et al. 2009). This is supported by the presence of 18 independent intron- 1 InDel mutations associated with a spring seasonal growth habit in wheat and barley (Cockram et al. 2007a, b; Fu et al. 2005; Dubcovsky et al. 2006; Hemming et al. 2009; Santra et al. 2009; Shcherban et al. 2012; von Zitzewitz et al. 2005). However, it should be noted that the influence of the VrnD1s allele on phenotype has not been formally defined here. According to passport data, the four accessions carrying the 


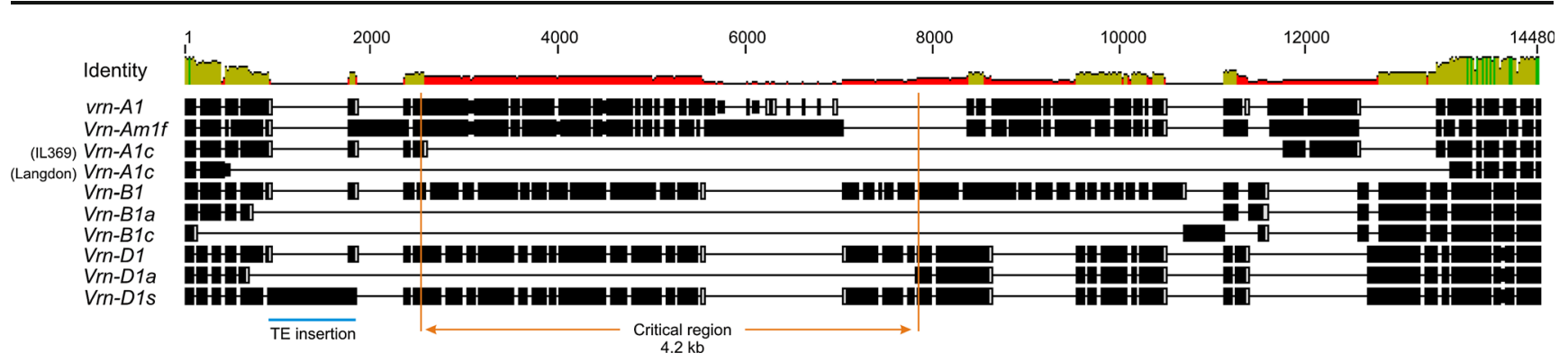

Fig. 4 Alignment of wheat $V r n 1$ intron-1 genomic DNA sequences. Alleles that carry large deletions (Vrn-Alc (type IL 369, Langdon), 5,504 and 7,222 bp; Vrn-B1a, 6,850 bp; Vrn-B1c, 6,850 and $820 \mathrm{bp}$;

TE insertion are all characterized as spring wheat. TE insertion in $V r n-D 1 s$ is located $87 \mathrm{bp}$ upstream from the TE insertions in $V r n-A m l f$ and $V r n-A m 1 h$. Further investigation of influence the TE insertion in $V r n-D l s$ on vernalization sensitivity will allow the borders of "critical region" to be established with greater precision.

Sequence analysis revealed the Vrn-D1 intron-1 insertion identified in this study represents a new non-autonomous transposable DNA element belonging to the $h A T$ superfamily. This superfamily includes the transposase-like genes that possess 8 bp TSDs and relatively short TIRs (5-27 bp) (Kempken et al. 2001), and mobilize via the classical "cut-and-paste" mechanism. Members of the $h A T$ superfamily have been observed in most eukaryotic lineages, including fungi, animals and plants (Rubin et al. 2001). The assumption that the TE identified here is non-autonomous is based on the following arguments: firstly, most autonomous $h A T$ family members include a transposase coding region with length of several thousands of kilobyte (Kempken et al. 2001). Secondly, multiple sequence alignment showed that central region 168-680 varies greatly between TEs with identical TIRs. Finally, similarity searches has not identified for this region of transposase encoding homologues.

According to the "80-80-80" rule (Wicker et al. 2007), two TEs belong to the same family if they share $80 \%$ (or more) sequence identity in $\geq 80 \%$ of their coding or internal domain, or within their terminal repeat regions, or in both. To avoid misclassification, it is recommended that segments $>80$ bp are analyzed. Therefore, compound or hybrid elements must be annotated segmentally, using minimum segment lengths of $80 \mathrm{bp}$ (Wicker et al. 2007). Following these conventions, similarity searches using the $5^{\prime}$ - and 3 '-TE regions in the $T$. aestivum genome showed that TIRs of associated with this transposon contain nonsynonymous SNP mutations. It is assumed that a characteristic of transposons from this family is a specific combination of 5'-TIR and 3'-TIR that are insignificantly different at specific sites. Compound transposons can arise from the juxtaposition of different individual TIRs or sequences resembling the TIRs of autonomous transposons (MacRae et al. 1992; Tsubota et al. 1991). This suggests that the TE identified here consists of remains (regions 1-167 and
$V r n-D 1 a, 4,235 \mathrm{bp})$ or TE insertion $\left(V r n-A^{m} 1 f, 493 \mathrm{bp}\right)$ associated with spring alleles are illustrated. The TE insertion in intron-1 of $V r n-D 1 s$ and the vernalization "critical region" are shown

681-836 bp) belonging to different transposons of closely related families. Furthermore, based on the strong conservation of TIRs, and their association with multiple TSDs, we conclude that the members of this TE family are involved in transposition.

Identification of the novel $V r n-D 1$ intron-1 TE insertion here will allow future studies to quantify its effect on vernalization response and early flowering, alone and in combination with spring alleles at the $V r n-A 1$ and/or $V r n-B 1$ loci. The diagnostic PCR assay for the presence of the $V r n-D 1$ allele developed here will help track this allele in future studies, and broaden the toolkit of diagnostic molecular assays available for $\mathrm{Vrn} 1$ alleles in temperate cereal species (Cockram et al. 2009; Fu et al. 2005; Shcherban et al. 2012; Yan et al. 2004a).

\section{References}

Anderson O, Dubcovsky J, Matthews D, Sabot F, Wicker T (2005) Nomenclature, classification and annotation of repetitive elements. http://wheat.pw.usda.gov/ITMI/Repeats/Nomenclatures_for_TREP. Accessed 4 December 2002, revised April 2005

Bariana H, Brown G, Ahmed N, Khatkar S, Conner R, Wellings C, Haley S, Sharp P, Laroche A (2002) Characterisation of Triticum vaviloviiderived stripe rust resistance using genetic, cytogenetic and molecular analyses and its marker-assisted selection. Theor Appl Genet 104(2-3):315-320

Bentley AR, Jensen EF, Mackay IJ, Hönicka H, Fladung M, Hori K, Yano M, Mullet JE, Armstead IP, Hayes C, Thorogood D, Lovatt A, Morris R, Pullen N, Mutasa-Göttgens E, Cockram J (2013) Flowering time. Genomics and breeding for climate-resilient crops 2 (Ed: C Cole):1-67

Brenchley R, Spannagl M, Pfeifer M, Barker GL, D'Amore R, Allen AM, McKenzie N, Kramer M, Kerhornou A, Bolser D, Kay S, Waite D, Trick M, Bancroft I, Gu Y, Huo N, Luo MC, Sehgal S, Gill B, Kianian S, Anderson O, Kersey P, Dvorak J, McCombie WR, Hall A, Mayer KF, Edwards KJ, Bevan MW, Hall N (2012) Analysis of the bread wheat genome using whole-genome shotgun sequencing. Nature 491(7426):705-710

Budowle B, Chakraborty R, Giusti AM, Eisenberg AJ, Allen RC (1991) Analysis of the VNTR locus D1S80 by the PCR followed by highresolution PAGE. Am J Hum Genet 48(1):137-144

Buerstmayr M, Lemmens M, Steiner B, Buerstmayr H (2011) Advanced backcross QTL mapping of resistance to Fusarium head blight and 
plant morphological traits in a Triticum macha $\times$ T. aestivum population. Theor Appl Genet 123(2):293-306

Chu CG, Tan CT, Yu GT, Zhong S, Xu SS, Yan L (2011) A novel retrotransposon inserted in the dominant $\mathrm{Vrn}-\mathrm{Bl}$ allele confers spring growth habit in tetraploid wheat (Triticum turgidum L.). G3 (Bethesda) 1(7):637-645

Cockram J, Chiapparino E, Taylor SA, Stamati K, Donini P, Laurie DA, O'sullivan DM (2007a) Haplotype analysis of vernalization loci in European barley germplasm reveals novel $V R N-H 1$ alleles and a predominant winter $V R N-H 1 / V R N-H 2$ multi-locus haplotype. Theor Appl Genet 115(7):993-1001

Cockram J, Mackay IJ, O'Sullivan DM (2007b) The role of doublestranded break repair in the creation of phenotypic diversity at cereal VRN1 loci. Genetics 177(4):2535-2539

Cockram J, Norris C, O'Sullivan DM (2009) PCR markers diagnostic for seasonal growth habit in barley. Crop Sci 49(2):403-410

Danyluk J, Kane N, Breton G, Limin A, Fowler D, Sarhan F (2003) TaVRT-1, a putative transcription factor associated with vegetative to reproductive transition in cereals. Plant Physiol 132(4): $1849-1860$

Diallo AO, Ali-Benali MA, Badawi M, Houde M, Sarhan F (2012) Expression of vernalization responsive genes in wheat is associated with histone $\mathrm{H} 3$ trimethylation. Mol Genet Genomics 287: $575-590$

Distelfeld A, Li C, Dubcovsky J (2009) Regulation of flowering in temperate cereals. Curr Opin Plant Biol 12(2):178-184

Doyle JJ, Doyle JL (1987) A rapid DNA isolation procedure for small quantities of fresh leaf tissue. Phytochemistry Bulletin 19:11-15

Dubcovsky J, Chen C, Yan L (2005) Molecular characterization of the allelic variation at the $\mathrm{VRN}-\mathrm{H} 2$ vernalization locus in barley. Mol Breed 15:395-407

Dubcovsky J, Loukoianov A, Fu D, Valarik M, Sanchez A, Yan L (2006) Effect of photoperiod on the regulation of wheat vernalization genes VRN1 and VRN2. Plant Mol Biol 60(64):469-480

Dubcovsky J, Li C, Distelfeld A, Pidal B, Tranquilli G (2008) Genes and gene networks regulating wheat development. In: Appels $\mathrm{R}$, Eastwood R, Lagudah E, Langridge P, Mackay M, McIntyre L, Sharp P (eds). Proceedings of 11th International Wheat Genetics Symposium: 24-29

Ferrandiz C, Gu Q, Martienssen R, Yanofsky MF (2000) Redundant regulation of meristem identity and plant architecture by FRUITFULL, APETALA1 and CAULIFLOWER. Development 127:725-734

Fu D, Szucs P, Yan L, Helguera M, Skinner J, Hayes P, Dubcovsky J (2005) Large deletions within the first intron in $V R N-1$ are associated with spring growth habit in barley and wheat. Mol Gen Genomics 273(4):54-65

Golovnina K, Kondratenko E, Blinov A, Goncharov N (2010) Molecular characterization of vernalization loci VRN1 in wild and cultivated wheats. BMC Plant Biology 10(168):168-183

Goncharov NP (2003) Genetics of growth habit (spring vs winter) in common wheat: confirmation of the existence of dominant $\operatorname{Vrn} 4$. Theor Appl Genet 107:768-772

Gultyaeva EI, Mikhailova LA, Karetnikova ES, Anpilova NA (2002) Triticum spelta $\mathrm{L}$. as a source of new genes of resistance to wheat leaf rust (Puccinia recondita Rob. ex Desm. f.sp. tritici). J Russ PhytopatholSoc 3:61-64

Hemming MN, Peacock WJ, Dennis ES, Trevaskis B (2008) Low temperature and day length cues are integrated to regulate FLOWERING LOCUS T in barley. Plant Physiol 147(1):355-366

Hemming MN, Fieg S, Peacock WJ, Dennis ES, Trevaskis B (2009) Regions associated with repression of the barley (Hordeum vulgare) VERNALIZATION1 gene are not required for cold induction. Mol Genet Genomics 282(2):107-117

Iwaki K, Nishida J, Yanagisawa T, Yoshida H, Kato K (2002) Genetic analysis of $V r n-B 1$ for vernalization requirement by using linked
dCAPS markers in bread wheat (Triticum aestivum L.). Theor Appl Genet 104(4):571-576

Kempken F, Windhofer F (2001) The hAT family: a versatile transposon group common to plants, fungi, animals, and man. Chromosoma 110(1):1-9

Kinjo H, Shitsukawa N, Takumi S, Murai K (2012) Diversification of three APETALA1/FRUITFULL-like genes in wheat. Mol Genet Genomics 287(4):283-294

Kippes N, Zhu J, Chen A, Vanzetti L, Lukaszewski A, Nishida H, Kato K, Dvorak J, Dubcovsky J (2014) Fine mapping and epistatic interactions of the vernalization gene $V R N-D 4$ in hexaploid wheat. Mol Genet Genomics 289(1):47-62

Kohany O, Gentles AJ, Hankus L, Jurka J (2006) Annotation, submission and screening of repetitive elements in Repbase: RepbaseSubmitter and Censor. BMC Bioinformatics 7:474-481

Law C, Worland A, Giorgi B (1975) The genetic control of ear emergence time by chromosomes 5A and 5D of wheat. Heredity 36(1):49-58

Liu J, He YH, Amasino R, Chen XM (2004) siRNAs targeting an intronic transposon in the regulation of natural flowering behavior in Arabidopsis. Genes Dev 18:2873-2878

Loukoianov A, Yan L, Blechl A, Sanchez A, Dubcovsky J (2005) Regulation of $V R N 1$ vernalization genes in normal and transgenic polyploid wheat. Plant Physiol 138(4):2364-2373

MacRae AF, Clegg MT (1992) Evolution of Ac and Ds1 elements in select grasses (Poaceae). Genetica 86:55-66

Nkongolo K, Quick J, Meyer W, Peairs F (1989) Russian wheat aphid resistance of wheat, rye and triticale in green-house tests. Cereal Res Commun 17:227-232

Oliver S, Finnegan E, Dennis E, Peacock W, Trevaskis B (2009) Vernalization-induced flowering in cereals is associated with changes in histone methylation at the VERNALIZATION1 gene. Proc Natl Acad Sci U S A 106(20):8386-8391

Pidal B, Yan L, Fu D, Zhang F, Tranquilli G, Dubcovsky J (2009) The CArG-Box located upstream from the transcriptional start of wheat vernalization gene $V R N 1$ is not necessary for the vernalization response. J Hered 100(3):355-364

Pugsley AT (1972) Additional genes inhabiting winter habit in wheat. Euphytica 21:547-552

Rubin E, Lithwick G, Levy AA (2001) Structure and evolution of the $h A T$ transposon superfamily. Genetics 158:949-957

Santra DK, Santra M, Allan RE, Campbell KG, Kidwell KK (2009) Genetic and molecular characterization of vernalization genes $\mathrm{Vrn}$ $A 1, V r n-B 1$, and $V r n-D 1$ in spring wheat germplasm from the pacific northwest region of the USA. Plant Breed 128:576-584

Shcherban AB, Efremova TT, Salina EA (2012) Identification of a new $V r n-B 1$ allele using two near-isogenic wheat lines with difference in heading time. Mol Breed 29(3):675-685

Shitsukawa N, Ikari C, Shimada S, Kitagawa S, Sakamoto K, Saito H, Ryuto H, Fukunishi N, Abe T, Takumi S, Nasuda S, Murai K (2007) The einkorn wheat (Triticum monococcum) mutant, maintained vegetative phase, is caused by a deletion in the VRN1 gene. Genes Genet Syst 82(2):167-170

Simon MR, Worland AJ, Cordo CA, Struik PC (2001) Chromosomal location of resistance to Septoria tritici in seedlings of a synthetic hexaploid wheat, Triticum spelta and two cultivars of Triticum aestivum. Euphytica 119(1):151-155

Steed A, Chandler E, Thomsett M, Gosman N, Faure S, Nicolson P (2005) Identification of type I resistance to Fusarium head blight controlled by a major gene located on chromosome 4A of Triticum macha. Theor Appl Genet 111:521-529

Stelmakh AF (1993) Genetic effects of Vrn genes on heading date and agronomic traits in bread wheat. Euphytica 65:53-60

Stelmakh AF (1998) Genetic systems regulating flowering response in wheat. Euphytica 100:359-369

Takumi S, Koyama K, Fujiwara K, Kobayashi F (2011) Identification of a large deletion in the first intron of the $\mathrm{Vrn}-\mathrm{D} 1 \mathrm{locus}$, associated with 
loss of vernalization requirement in wild wheat progenitor Aegilops tauschii Coss. Genes Genet Syst 86(3):183-195

Thompson JD, Higgins DG, Gibson TJ (1994) CLUSTAL W: improving the sensitivity of progressive multiple sequence alignment through sequence weighting, position-specific gap penalties and weight matrix choice. Nucleic Acids Res 22:4673-4680

Trevaskis B, Bagnall D, Ellis M, Peacock W, Dennis E (2003) MADS box genes control vernalization-induced flowering in cereals. Proc Natl Acad Sci U S A 100(22):13099-13104

Trevaskis B, Hemming M, Dennis E, Peacock W (2007) The molecular basis of vernalization-induced flowering in cereals. Trends Plant Sci 12(8):352-357

Tsubota SI, Huong DV (1991) Capture of flanking DNA by a P element in Drosophila melanogaster: creation of a transposable element. Proc Natl Acad Sci U S A 88:693-697

von Zitzewitz J, Szucs P, Dubcovsky J, Yan L, Francia E, Pecchioni N, Casas A, Chen TH, Hayes PM, Skinner JS (2005) Molecular and structural characterization of barley vernalization genes. Plant Mol Biol 59(3):449-467

Wicker T, Sabot F, Hua-Van A, Bennetzen JL, Capy P, Chalhoub B, Flavell A, Leroy P, Morgante M, Panaud O, Paux E, SanMiguel P,
Schulman AH (2007) A unified classification system for eukaryotic transposable elements. Nat Rev Genet 8(12):973-982

Yan L, Loukoianov A, Tranquilli G, Helguera M, Fahima T, Dubcovsky J (2003) Positional cloning of the wheat vernalization gene VRN1. Proc Natl Acad Sci U S A 100(10):6263-6268

Yan L, Helguera M, Kato K, Fukuyama S, Sherman J, Dubcovsky J (2004a) Allelic variation at the VRN1 promoter region in polyploid wheat. Theor Appl Genet 109(8):1677-1686

Yan L, Loukoianov A, Blechl A, Tranquilli G, Ramakrishna W, SanMiguel P, Bennetzen J, Echenique V, Dubcovsky J (2004b) The wheat VRN2 gene is a flowering repressor down-regulated by vernalization. Science 303(5664):1640-1644

Yan L, Fu D, Li C, Blechl A, Tranquilli G, Bonafede M (2006) The wheat and barley vernalization gene VRN3 is an orthologue of FT. Proc Natl Acad Sci U S A 103(51):19581-19586

Zhang Z, Schwartz S, Wagner L, Miller W (2000) A greedy algorithm for aligning DNA sequences. J Comput Biol 7(1-2):203-214

Zhang J, Wang Y, Wu S, Yang J, Liu H, Zhou Y (2012) A single nucleotide polymorphism at the $\mathrm{Vrn}-\mathrm{Dl}$ promoter region in common wheat is associated with vernalization response. Theor Appl Genet 125(8):1697-1704 